\title{
Optimal Compliance with Emission Constraints \\ Dynamic Characteristics and the Choice of Technique
}

\section{Journal Article}

Author(s):

Winkler, Ralph

Publication date:

2008

Permanent link:

https://doi.org/10.3929/ethz-b-000010952

Rights / license:

In Copyright - Non-Commercial Use Permitted

Originally published in:

Environmental and Resource Economics 39(4), https://doi.org/10.1007/s10640-007-9133-4 


\title{
Optimal compliance with emission constraints: dynamic characteristics and the choice of technique
}

\author{
Ralph Winkler
}

Received: 11 July 2005 / Accepted: 13 April 2007 / Published online: 15 June 2007

(C) Springer Science+Business Media B.V. 2007

\begin{abstract}
The paper analyzes how to comply with an emission constraint, which restricts the use of an established energy technique, given the two options to save energy and to invest in two alternative energy techniques. These techniques differ in their deterioration rates and the investment lags of the corresponding capital stocks. Thus, the paper takes a medium-term perspective on climate change mitigation, where the time horizon is too short for technological change to occur, but long enough for capital stocks to accumulate and deteriorate. It is shown that, in general, only one of the two alternative techniques prevails in the stationary state, although, both techniques might be utilized during the transition phase. Hence, while in a static economy only one technique is efficient, this is not necessarily true in a dynamic economy.
\end{abstract}

Keywords Climate change mitigation - Dynamic characteristics - Emission targets · Environmental policy - Optimal structural change - Replacement of energy techniques · Static versus dynamic efficiency · Time-lagged dynamic optimization

JEL-Classification Q48 - Q53 · C61

\section{Introduction}

Many environmental problems, humankind faces today, are of a long-run nature. A prime example is anthropogenic climate change, which is caused by the emissions of so-called greenhouse gases. These gases, in particular $\mathrm{CO}_{2}$, exhibit long life times in the atmosphere and, thus, accumulate over time and incur a long lasting effect on global climatic conditions. In industrialized countries, the biggest sources of $\mathrm{CO}_{2}$ emissions are the combustion of fossil fuels for energy production and transportation. Therefore, it is clear that a successful climate change mitigation strategy has to include a shift from carbon intensive to carbon neutral

R. Winkler $(\bowtie)$

CER-ETH-Center of Economic Research at ETH Zurich, ZUE D4, 8092 Zurich, Switzerland

E-mail: mail@ralph-winkler.de 
energy technologies in the long run. In this long-run perspective, however, the set of technologies is not constant, but changes over time. New technologies are invented and (partly) replace formerly established technologies. Thus, a better understanding of the causes and determinants for technological change are inevitable for the long-run solution of anthropogenic climate change.

Complimentary to such a long-run approach on climate change mitigation, is a short-run point of view. In this perspective, the question of "burden sharing", i.e., which country cuts emissions of greenhouse gases by how much, dominates over the long-run aspects of stock pollutant accumulation and technological change. A prime example for such a burden sharing treaty is the Kyoto protocol (United Nations 1997). Therein, most industrialized countries committed to reduce their $\mathrm{CO}_{2}$ emissions in the period from 2008-2012 by an average of 5\% compared to the emissions in 1990. As it is doubted that the Kyoto protocol target would have a significant impact on mitigating global climate change (for a review and critical assessment see, e.g., Böehringer 2003), some countries have even committed to more drastic emission reductions. The German government, for example, adopted $1995 \mathrm{a} \mathrm{CO}_{2}$ reduction target of $25 \%$ by 2005 , compared to the emissions in 1990 .

In this paper, I focus on a time horizon, which lies between the short-run and the longrun point of view. In such a medium-term perspective, a burden sharing treaty, such as the Kyoto protocol, is already agreed on. Moreover, I assume that the time horizon of this treaty is too short for technological change to occur, but long enough for the accumulation of technology specific capital stocks of already known and available production techniques. As a consequence, emission reductions have to be achieved by a structural change of the economy, either by increasing energy efficiency and reducing energy intensive consumption or by replacing fossil fuel-based energy techniques by already available alternative energy producing techniques.

In particular, I analyze how an economy optimally complies with the introduction of a binding emission constraint, which restricts the use of an established energy producing technique. In accordance with a medium-term perspective, I do not consider explicitly the long-run environmental damage caused by the stock accumulating property of greenhouse gases, and I also abstract from technological change and growth. Yet, the economy has two options to comply with the emission constraint. First, it can save energy by transferring labor from the established energy technique to consumption good production. Second, the established energy technique can be partly replaced by two already known and available alternative energy producing techniques. Each of the two linear-limitational alternative techniques combine labor and a specific capital good, which takes a certain time span to produce (i.e., exhibits an investment lag) and which deteriorates at a certain rate. These two dynamic characteristics, investment lag and deterioration rate, are considered to be different for the two alternative techniques. In particular, I am interested in how the optimal transition toward the new (medium-term) stationary state takes place, dependent on the dynamic characteristics of the alternative energy producing techniques.

The economic literature has addressed both the long-run and the short-run perspective on the mitigation of climate change. For the long-term perspective, the induced technological change debate plays an important role. ${ }^{1}$ The intertemporal nature of the climate change problem is addressed either in an endogenous growth framework or in an integrated assess-

\footnotetext{
${ }^{1}$ Other relevant, but more general issues in the case of the long-term perspective on climate change mitigation include accumulation of stock pollutants (e.g., Aaheim 1999; Falk and Mendelsohn 1993; Moslener and Requate forthcoming), uncertainty and irreversibility (e.g., Gollier and Treich 2003; Kolstad 1996; Lange 2003; Newell and Pizer 2003; Ulph and Ulph 1997), and discounting and intergenerational equity (e.g., Karp 2005; Li and Löfgren 2000; Lind 1982; Chichilnisky 1996; Portney and Weyant 1999; Weitzman 1998).
} 
ment model by scenario simulation. In the mostly used bottom-up approaches, induced technological change is studied by applying one representative aggregated production technology, which becomes more efficient and/or less polluting by technological change (e.g., Goulder and Mathai 2000; Nordhaus 2002; Newell et al. 1999). In the top-down approaches, induced technological change also allows for structural change between a set of competing technologies (e.g., Gerlagh and Van der Zwaan 2003; Goulder and Schneider 1999; Van der zwaan et al. 2002). However, the impact of induced technological change on climate change mitigation varies greatly among the different studies (e.g., Carraro et al.2003; Löeschel 2002; Gerlagh and Lise 2005). Another approach to the analysis of induced technological change explicitly allows for the strategic interaction between the regulator, an innovating R\&D sector and firms which adopt new technology (e.g., Fisher et al. 2003; Laffont and Tirole 1996; Montero 2002; Phaneuf and Requate 2002; Requate and Unold 2003; Requate forthcoming).

One strand of the literature, which addresses the short-run perspective, analyzes the effectiveness of different policy instruments to reduce emissions, either in theoretical (e.g., Aidt and Dutta 2004; Hoel and Karp 2000; Montero 2002; Pezzey 2003) or in computational general equilibrium (CGE) models (e.g., Böehringer and Vogt 2003; Buonanno et al. 2003; Dellink et al. 2004; Pizer 2002). Another strand of the literature considers strategic interactions between different countries, which negotiate emission reductions (e.g., Endres and Finus 1999, 2002; Rubio and Casino 2005; Yang 2003), or analyze optimal policy instruments with strategic interactions between a regulator and individual firms (e.g., Gersbach and Glazer 1999; Gersbach and Requate 2004; Moledina et al. 2003).

Despite this extensive literature on the long-run and the short-run perspective of climate change mitigation, there is a lack of literature on the medium-term, where the agreement on the burden sharing treaty is already achieved and has to be implemented by the set of already existing energy saving options and alternative energy producing techniques. The aim of this paper is to provide (to the very best of my knowledge) the first theoretical analysis of this medium-term perspective on climate change mitigation and the corresponding choice of techniques based on their dynamic characteristics. ${ }^{2}$

Therefore, I combine emission constraints with heterogeneous time-lagged capital theory. ${ }^{3}$ I show that it might be optimal to utilize both techniques on the transition paths toward the new stationary state, although, in general, only one of the two alternative techniques prevails in the (medium-term) stationary state (and only if replacement is superior to energy saving alone). Hence, while in a static economy only one technique is efficient, this is not necessarily true in a dynamic economy. Although this paper is predominantly a theoretical discussion of a specific problem (i.e., the optimal compliance with an emission target in a medium-term perspective), the conclusions point toward a more general applicability, which has to be explicitly investigated in future research.

The paper is organized as follows. Section 2 introduces the model. The resulting intertemporal optimization problem and its necessary and sufficient conditions are discussed in Sect. 3. In Sect. 4, the different stationary state equilibria are discussed, while Sect. 5 is devoted to the optimal transition path. In Sect. 6, the underlying model assumptions are critically reviewed and some policy implications are discussed. Sect. 7 concludes.

\footnotetext{
2 Therefore, my paper is somewhat related to Bhaduri (1968) and Nuti (1970), who also draw attention on what I call dynamic characteristics, however, in a completely different context.

3 The time-lagged accumulation of capital is an idea which dates back to the Austrian school of economics (von Böehm-Bawerk [1889]1921), and was revived by the neo-Austrian capital theory in the 1970s (e.g., von Weizsäecker 1971; Hicks 1973; Faber 1979). Most recently, it has been discussed in the macroeconomics real business cycle literature (e.g., Kydland and Prescott 1982; Ioannides and Taub 1992) and in the vintage capital growth theory (e.g., Boucekkine et al. 2005; Feichtinger et al. 2006).
} 


\section{The model}

In this section, I introduce a simple dynamic model to analyze the optimal compliance of an economy to an exogenously given emission target. For reasons of tractability, emphasis is laid on a most simplistic model structure, which is still rich enough to capture the dynamics of the medium-term perspective as outline in Sect. 1.

Consider an economy with a single primary input factor (e.g., labor) given in the constant amount $\bar{l}$ at all times, which is distributed among six production processes within three different production sectors: the energy sector, the consumption good sector, and the investment sector.

The energy sector comprises three different energy producing techniques, represented by three linear-limitational production processes. The rationale for using Leontief-type production functions is (apart from tractability considerations) to account for rigidities in energy production due to technical and thermodynamical constraints. However, for the sake of simplicity, fuel inputs are not considered explicitly.

The first process, the status quo process in the economy, is considered to produce energy solely by means of labor: ${ }^{4}$

$$
x_{0}(t)=l_{0}(t) .
$$

In addition, the first process is considered to jointly produce an unwanted and, at least potentially, harmful pollutant. The emissions $e$ are proportional to the amount of energy $x_{0}$ produced:

$$
e(t)=x_{0}(t)=l_{0}(t) .
$$

From time $t=0$ onward, a binding emission constraint is enacted to reduce the emissions $e$ of the pollutant jointly produced by the status quo energy producing technique:

$$
x_{0}(t)=l_{0}(t) \leq \phi .
$$

Furthermore, the economy has access to two 'clean' energy producing techniques, which do not jointly produce a pollutant. Each of these alternative processes utilizes a special capital good in a Leontief-type production function. Thus, one unit of energy is produced by combining $\lambda_{h}$ units of labor together with $\kappa_{h}$ units of capital $(h=1,2)$ :

$$
x_{h}(t)=\min \left[\frac{l_{h}(t)}{\lambda_{h}}, \frac{k_{h}(t)}{\kappa_{h}}\right], \quad h=1,2 .
$$

For the remainder of the paper, I refer to the Energy Producing Techniques (1) and (4) as $\mathrm{EPT}_{0}, \mathrm{EPT}_{1}$, and $\mathrm{EPT}_{2}$.

Energy is considered to be homogeneous and non-storable. As a consequence, the total energy $x$ available at time $t$ equals:

$$
x(t)=x_{0}(t)+x_{1}(t)+x_{2}(t) .
$$

The specific capital goods $k_{h}$ necessary to produce energy via $\mathrm{EPT}_{h}(h=1,2)$, are produced in the investment sector. For the sake of simplicity, the production of the specific capital goods

\footnotetext{
4 Neglecting capital in the status quo technology is justified because of two reasons. First, the status quo technique is assumed to be fully developed at the beginning of the time horizon. Second, as capital is considered to be specific to the different production processes, it cannot be moved from the status quo to the alternative techniques. Hence, capital in the status quo technique need not to be considered explicitly, as there is no inertia from building up the capital stock and the capital stock is sunk, once it cannot be fully employed anymore.
} 
is assumed to take the following form: one unit of labor input equals one unit of investment in the specific capital goods.

$$
i_{h}(t)=l_{h+2}(t), \quad h=1,2 .
$$

However, the investment $i_{h}$ is considered to take the time span $\sigma_{h}$ until it turns into productive capital $k_{h}(h=1,2)$. Furthermore, the capital goods $k_{h}$ deteriorate at the constant and positive rates $\gamma_{h}$. Hence, the equations of motion for the two capital stocks read:

$$
\dot{k}_{h}(t)=i_{h}\left(t-\sigma_{h}\right)-\gamma_{h} k_{h}(t), \quad h=1,2 .
$$

The intuition behind these assumptions is that energy plants are not built in a day. In fact, energy plants need considerable time to build. Moreover, both the time to built and the deterioration rate are technology specific and vary greatly among different energy technologies. Hence, each alternative energy producing technique is characterized by two dynamic characteristics: the investment period $\sigma_{h}$ and the deterioration rate $\gamma_{h}(h=1,2)$. Without loss of generality, I assume $\sigma_{1}<\sigma_{2}$.

The consumption good sector comprises one aggregated Cobb-Douglas production function, which allows for the substitution between labor $l$ and energy $x$ :

$$
c(t)=F(l(t), x(t))=l(t)^{\alpha} x(t)^{1-\alpha}, \quad 0>\alpha>1 .
$$

The intuition behind the choice of a Cobb-Douglas production function for the consumption good, while the energy producing techniques are linear-limitational, is twofold. First, in the model the consumption good sector is more aggregated than the energy sector, as it is only represented by one production technique, compared to three energy producing techniques. Thus, if one considers an internal structure of the consumption good sector, there is the possibility of substituting labor and energy by employing different ratios of the microeconomic production techniques. Second, while energy is a homogeneous commodity, consumption is not. Thus, society can substitute energy by labor, by producing less energy intensive consumption goods.

At the beginning of the time horizon, $\mathrm{EPT}_{0}$ is considered to be the status quo in the economy. Hence, the economy has not utilized the two alternative energy producing technologies in the past. Note that the economy has two options how to comply with the emission constraint $\phi$. The labor, freed from $\mathrm{EPT}_{0}$ due to the emission constraint, can either be used to produce energy by the clean techniques $\mathrm{EPT}_{1}$ and $\mathrm{EPT}_{2}$, or energy can be saved by employing the surplus labor directly in the consumption good process (8).

In order to decide how to comply with the emission constraint, society seeks to maximize the intertemporal welfare functional $W$ of a representative consumer

$$
W\{c(t)\}=\int_{0}^{\infty} V(c(t)) \exp [-\rho t] \mathrm{d} t,
$$

where $V$ is a twice continuously differentiable, strictly increasing and concave instantaneous welfare function $\left(V_{c}>0, V_{c c}<0\right)$, depending solely on consumption $c$, and $\rho$ is the constant and positive discount rate. Thus, emissions do not directly influence welfare, and environmental concerns are solely reflected by the introduction of the emission constraint. This is justified, if costs of environmental protection occur today, while benefits occur in the distant future and/or are uncertain and, therefore, difficult to quantify. ${ }^{5}$

5 In the terminology of Winkler (2005), this approach represents the ecological solution to incorporate joint production in welfare analysis. 
In the following, I analyze whether and, if so, which of the two alternative energy producing techniques, $\mathrm{EPT}_{1}$ and $\mathrm{EPT}_{2}$, partly replaces the status quo technique, in order to comply with the emission constraint $\phi$. Moreover, I am interested not only in the (mediumterm) stationary state, but also in the transition dynamics. Obviously, both of these hinge, in general, upon the functional form of the instantaneous welfare function $V$, the exogenously given parameters $\alpha, \lambda_{1}, \lambda_{2}, \kappa_{1}, \kappa_{2}, \rho$, and $\phi$, and especially upon the dynamic characteristics $\sigma_{1}, \sigma_{2}, \gamma_{1}$, and $\gamma_{2}$ of the two alternative techniques.

\section{The intertemporal optimization problem}

As welfare is strictly increasing in consumption, and consumption good production can always be increased by assigning more labor to the consumption good process (8), the labor restriction will hold with equality along the optimal path:

$$
l(t)=\bar{l}-l_{0}(t)-l_{1}(t)-l_{2}(t)-l_{3}(t)-l_{4}(t) .
$$

Assuming efficient production of $\mathrm{EPT}_{1}$ and $\mathrm{EPT}_{2}$, i.e., $l_{h}(t) / \lambda_{h}=k_{h}(t) / \kappa_{h}(h=1,2)$, and inserting Eq. 6 into the labor restriction (10), yields:

$$
l(t)=\bar{l}-l_{0}(t)-\frac{\lambda_{1}}{\kappa_{1}} k_{1}(t)-\frac{\lambda_{2}}{\kappa_{2}} k_{2}(t)-i_{i}(t)-i_{2}(t) .
$$

Analogously, by inserting Eqs. 1 and 4 into Eq. 5, one derives for total energy production $x$ :

$$
x(t)=l_{0}(t)+\frac{k_{1}(t)}{\kappa_{1}}+\frac{k_{2}(t)}{\kappa_{2}} .
$$

Hence, consumption $c$ is a function of the control variables $l_{0}, i_{1}$, and $i_{2}$, and the stock variables $k_{1}$ and $k_{2}$ :

$$
\begin{aligned}
c(t)=F(l(t), x(t))= & {\left[\bar{l}-l_{0}(t)-\frac{\lambda_{1}}{\kappa_{1}} k_{1}(t)-\frac{\lambda_{2}}{\kappa_{2}} k_{2}(t)-i_{1}(t)-i_{2}(t)\right]^{\alpha} } \\
& \times\left[l_{0}(t)+\frac{k_{1}(t)}{\kappa_{1}}+\frac{k_{2}(t)}{\kappa_{2}}\right]^{1-\alpha} .
\end{aligned}
$$

Assuming that investment is non-negative, i.e., the capital stocks cannot be consumed, the intertemporal optimization problem, society seeks to solve, reads:

$$
\max _{l_{0}, i_{1}, i_{2}} \int_{0}^{\infty} V(c(t)) \exp [-\rho t] \mathrm{d} t
$$

subject to

$$
\begin{aligned}
c(t) & =F(l(t), x(t)), \\
\dot{k}_{h}(t) & =i_{h}\left(t-\sigma_{h}\right)-\gamma_{h} k_{h}(t), \quad h=1,2, \\
i_{h}(t) & \geq 0, \quad h=1,2, \\
\phi & \geq l_{0}(t), \\
i_{h}(t) & =\xi_{h}(t)=0, \quad t \in\left[-\sigma_{h}, 0\right), \quad h=1,2, \\
k_{h}(0) & =0, \quad h=1,2 .
\end{aligned}
$$

Because of the positive time-lags $\sigma_{h}(h=1,2)$, the equations of motion for the capital stocks $k_{h}$ become retarded differential-difference equations. Thus, variations in the capital 
stock $k_{h}$ not only depend on parameters evaluated at time $t$, but also on parameters evaluated at the earlier time $t-\sigma_{h}$. As a consequence, and in contrast to instantaneous accumulation models, the specification of an initial value $k_{h}(0)$ for the capital stock $k_{h}$ is not sufficient for a unique solution. In addition, an initial path $\xi_{h}$ for the investment $i_{h}$ in the time interval $\left[-\sigma_{h}, 0\right)$ has to be specified. The path of the capital stock $k_{h}$ in the time interval $t \in\left[0, \sigma_{h}\right]$ is completely determined by the initial capital stock $k_{h}(0)$, the initial path $\xi_{h}$, and the retarded equation of motion. Thus, time-lagged accumulation models exhibit an additional moment of inertia, as the capital stocks react with a delay to variations in investment.

\subsection{Necessary and sufficient conditions of the optimal solution}

To solve the resulting optimization problem, I apply the generalized maximum principle derived in El-Hodiri et al. (1972) for time-lagged optimal control problems. One obtains the following present-value Hamiltonian $\mathcal{H}$

$$
\begin{aligned}
\mathcal{H}= & V(c(t)) \exp [-\rho t]+p_{c}(t)[F(l(t), x(t))-c(t)] \\
& +p_{k_{1}}\left(t+\sigma_{1}\right) i_{1}(t)-p_{k_{1}}(t) \gamma_{1} k_{1}(t)+p_{k_{2}}\left(t+\sigma_{2}\right) i_{2}(t)-p_{k_{2}}(t) \gamma_{2} k_{2}(t) \\
& +p_{i_{1}}(t) i_{1}(t)+p_{i_{2}}(t) i_{2}(t)+p_{\phi}(t)\left[\phi-l_{0}(t)\right],
\end{aligned}
$$

where $p_{c}, p_{i_{1}}, p_{i_{2}}$, and $p_{\phi}$ denote the Kuhn-Tucker parameters for the corresponding constraints, and $p_{k_{1}}$ and $p_{k_{2}}$ are the costate variables for the capital stocks $k_{1}$ and $k_{2}$. The difference from instantaneous capital accumulation models is covered by the term $p_{k_{h}}\left(t+\sigma_{h}\right) i_{h}(t)$ $(h=1,2){ }^{6}$

Assuming that the Hamiltonian $\mathcal{H}$ is continuously differentiable with respect to $i_{h}(h=$ $1,2)$, the following necessary conditions hold for an optimal solution (partial derivatives of the functions $\mathcal{H}, V$, and $F$ are indicated by subscripts and only the time argument is stated explicitly):

$$
\begin{aligned}
\mathcal{H}_{c} & =V_{c}(t) \exp [-\rho t]-p_{c}(t)=0, \\
\mathcal{H}_{l_{0}} & =p_{c}(t)\left[F_{x}(t)-F_{l}(t)\right]-p_{\phi}(t)=0, \\
\mathcal{H}_{i_{h}} & =-p_{c}(t) F_{l}(t)+p_{k_{h}}\left(t+\sigma_{h}\right)+p_{i_{h}}(t)=0, \quad h=1,2, \\
\mathcal{H}_{k_{h}} & =p_{c}(t)\left[\frac{1}{\kappa_{h}} F_{x}(t)-\frac{\lambda_{h}}{\kappa_{h}} F_{l}(t)\right]-\gamma_{h} p_{k_{h}}(t)=-\dot{p}_{k_{h}}(t), \quad h=1,2, \\
p_{i_{h}}(t) & \geq 0, \quad p_{i_{h}}(t) i_{h}(t)=0, \quad h=1,2, \\
p_{\phi}(t) & \geq 0, \quad p_{\phi}(t)\left[\phi-l_{0}(t)\right]=0 .
\end{aligned}
$$

Due to the concavity of the Hamiltonian $\mathcal{H}$, the necessary conditions are also sufficient, if, in addition, the following transversality conditions hold:

$$
\lim _{t \rightarrow \infty} p_{k_{h}}(t) k_{h}(t)=0, \quad h=1,2 .
$$

The economic interpretation of the necessary and sufficient conditions is straightforward. Condition (16a) says that, along the optimal path, the shadow price of the consumption good equals the marginal utility of consumption. According to conditions (16b) and (16f), $p_{\phi}=0$ and $F_{x}$ equals $F_{l}$, if the emission constraint is not binding. In this case, society is indifferent,

\footnotetext{
6 Although it might look odd at first sight to have $p_{k_{h}}(h=1,2)$ evaluated at a future time, while there are retarded equations of motion $(14 \mathrm{c})$, the explanation is quite intuitive: $p_{k_{h}}$ measures the net present value of all future welfare gains of one additional unit of capital $k_{h}$. As investment takes the time period $\sigma_{h}$ to turn into productive capital, the investment $i_{h}(t)$ gives rise to additional capital $k_{h}$ at $t+\sigma_{h}$, the net present value of which is given by $p_{k_{h}}\left(t+\sigma_{h}\right)$.
} 
whether to apply one additional marginal unit of labor directly to the consumption good process, or use it to produce an additional marginal unit of energy, which is then applied in the consumption good process. If the emission constraint is binding, $p_{\phi}$ is non-negative and measures the welfare gain society would be able to achieve, by taking one marginal unit of labor from the consumption good process (8) and apply it to the production of energy in $\mathrm{EPT}_{0}$.

Together with the transversality conditions (16g), conditions (16d) can be unambiguously solved to yield:

$$
p_{k_{h}}(t)=\int_{t}^{\infty} p_{c}\left(t^{\prime}\right)\left[\frac{1}{\kappa_{h}} F_{x}\left(t^{\prime}\right)-\frac{\lambda_{h}}{\kappa_{h}} F_{l}\left(t^{\prime}\right)\right] \exp \left[-\gamma_{h}\left(t^{\prime}-t\right)\right] \mathrm{d} t^{\prime}, \quad h=1,2 .
$$

Hence, in the optimum the shadow prices for the capital stocks equal the net present value of all future welfare gains of one additional marginal unit of the corresponding capital good. As capital goods are long lived, they contribute over the whole time horizon, although, increasingly less due to deterioration. The term in square brackets gives the marginal productivity of an additional unit of labor. On the one hand, an additional unit of capital drains labor from the consumption good process, in order to be employed. On the other hand, the capital good produces additional energy, which increases consumption good production.

The remaining conditions, (16c) and (16e), imply that, as long as $p_{i_{h}}=0$ and, thus, $i_{h} \geq 0(h=1,2)$, the welfare loss of the investment of an additional marginal unit has to equal the net present value of the future welfare gains of an additional marginal unit of capital. As investment needs time to turn into productive capital, the shadow prices of capital are evaluated at the future time $t+\sigma_{h}$.

For an investment in both alternative energy producing techniques to be optimal over the whole time horizon, $p_{i_{1}}=0$ and $p_{i_{2}}=0$ have to hold for all $t$. According to the conditions (16c) this is equivalent to $p_{k_{1}}\left(t+\sigma_{1}\right)=p_{k_{2}}\left(t+\sigma_{2}\right)$ for all $t$. This also implies that $\dot{p}_{k_{1}}\left(t+\sigma_{1}\right)=\dot{p}_{k_{2}}\left(t+\sigma_{2}\right)$ for all $t$. Hence, equating (16d) for $h=1$, and $h=2$ yields:

$$
\frac{\kappa_{2} \exp \left[\rho \sigma_{2}\right]}{\kappa_{1} \exp \left[\rho \sigma_{1}\right]}=\frac{V_{c}\left(t+\sigma_{2}\right)\left[F_{x}\left(t+\sigma_{2}\right)-\left(\lambda_{2}+\kappa_{2} \gamma_{2}\right) F_{l}\left(t+\sigma_{2}\right)\right]}{V_{c}\left(t+\sigma_{1}\right)\left[F_{x}\left(t+\sigma_{1}\right)-\left(\lambda_{1}+\kappa_{1} \gamma_{1}\right) F_{l}\left(t+\sigma_{1}\right)\right]} .
$$

As $c(t), l(t)$, and $x(t)$ change over time, and $V$ is a generic function, this equation can only hold accidentally for all $t$. Thus, in general, only one of the two alternative energy producing techniques will partly replace the status quo technique in the (medium-term) stationary state.

\subsection{Restrictions on exogenous parameters}

I further structure the optimization problem (14) by imposing the following two assumptions:

Assumption 1 (binding emission constraint) The emission constraint is binding, i.e., the emission constraint is smaller than the emissions would be without an emission constraint.

Assumption 2 (inefficiency of alternative techniques) Society evaluates the alternative techniques to be inferior compared to the status quo technique, if the emissions are not taken into account, i.e., it is not optimal to replace $\mathrm{EPT}_{0}$ by $\mathrm{EPT}_{1}$ and/or $\mathrm{EPT}_{2}$ without the enactment of a binding emission constraint.

These assumptions impose restrictions on the exogenously given parameters. As shown in the appendix, Assumption 1 implies that

$$
\phi \in\left[0, e^{\star}\right), \quad e^{\star}=(1-\alpha) \bar{l} .
$$


Thus, it is convenient to express the emission constraint as a fraction $\psi$ of the status quo emissions:

$$
\phi=\psi e^{\star}, \quad \psi \in(0,1) .
$$

For Assumption 2 to hold the following inequalities have to hold simultaneously (proof in the appendix):

$$
1 \leq \lambda_{h}+\kappa_{h}\left(\rho+\gamma_{h}\right) \exp \left[\rho \sigma_{h}\right], \quad h=1,2 .
$$

The conditions (21) say that, if the welfare costs (measured in units of labor) of the production of one unit of energy are smaller for $\mathrm{EPT}_{0}$ (left-hand-side) than for the alternative techniques $\mathrm{EPT}_{h}$ (right-hand-side of conditions (21)), then $\mathrm{EPT}_{h}$ will not be used without a binding emission constraint $(h=1,2)$.

The optimization problem (14) cannot be solved analytically. Thus, the straightforward approach, to solve the optimization problem in order to determine the optimal transition path, is not applicable. However, it is possible to analytically derive the (medium-term) stationary state and some qualitative properties of the optimal solution.

\section{Stationary state equilibria}

Although conditions (20) and (21) restrict the possible outcomes of the optimization problem (14), there are still two qualitatively different stationary state equilibria for the economy. (1) $\mathrm{EPT}_{1}$ and $\mathrm{EPT}_{2}$ can be so inferior compared to $\mathrm{EPT}_{0}$ that it is optimal to use the labor, which cannot be employed anymore in $\mathrm{EPT}_{0}$ because of the binding emission constraint, directly in the consumption good process (8) instead of utilizing EPT 1 or EPT 2 . (2) At least one of the two alternative techniques is so efficient that its use is optimal in the presence of a binding emission constraint. Then, $\mathrm{EPT}_{0}$ is partly replaced by $\mathrm{EPT}_{1}$ and/or $\mathrm{EPT}_{2}$.

\subsection{Optimal compliance by energy saving alone}

In the first case, the binding emission constraint is reached by energy saving alone. The conditions for the exogenously given parameters for energy saving alone to be optimal are given by the following proposition.

Proposition 1 (energy saving only) Given the optimization problem (14) and a binding emission constraint (20), it is optimal to employ all labor, which is freed by the binding emission constraint $\phi$, in the consumption good process (8) and neither utilize $\mathrm{EPT}_{1}$ or $\mathrm{EPT}_{2}$, if the following condition holds simultaneously for $h=1$ and $h=2$ :

$$
1+\frac{1-\psi}{\alpha \psi} \leq \lambda_{h}+\kappa_{h}\left(\rho+\gamma_{h}\right) \exp \left[\rho \sigma_{h}\right], \quad h=1,2 .
$$

Proof In the appendix.

If inequality (22) holds simultaneously for $h=1$, and $h=2$, society will not invest in either of the two alternative energy producing technologies and remain in the stationary state given by (A.4). In this case, the emission constraint is reached by energy saving alone, i.e., the labor freed from $\mathrm{EPT}_{0}$ is directly used in the consumption good process (8). 
4.2 Optimal compliance by replacement of energy techniques

If at least one of the inequalities (22) is violated, energy saving alone, in order to comply with the emission constraint, is not optimal. In fact, society will invest at least in one of the alternative techniques $\mathrm{EPT}_{1}$ or $\mathrm{EPT}_{2}$. As a consequence, the economy will not stay in a stationary state, as in the case of energy saving alone, but follow a dynamic transition path toward a new stationary state, where the status quo technique $\mathrm{EPT}_{0}$ is partly replaced by $\mathrm{EPT}_{1}$ or $\mathrm{EPT}_{2}$. In general, only one of the two alternative energy producing techniques, $\mathrm{EPT}_{1}$ or $\mathrm{EPT}_{2}$, prevails in this new stationary state, as the following proposition states.

Proposition 2 (replacement of energy techniques) Given the optimization problem (14), a binding emission constraint (20) and further assuming that condition (22) is violated for both $h=1$, and $h=2$, the alternative energy producing technique with the lower unit costs of energy prevails in the stationary state, i.e., $\lambda_{h}+\kappa_{h}\left(\rho+\gamma_{h}\right) \exp \left[\rho \sigma_{h}\right](h=1,2)$. Assuming that $\mathrm{EPT}_{h}(h=1,2)$ has the lower unit costs of energy, the stationary state is given by the following equations:

$$
\begin{aligned}
l_{0}^{\star}= & \bar{l}(1-\alpha) \psi, \\
i_{h}^{\star}= & \bar{l}(1-\alpha) \gamma_{h} \kappa_{h} A_{h}, \\
k_{h}^{\star}= & \bar{l}(1-\alpha) \kappa_{h} A_{h}, \\
l^{\star}= & \bar{l}\left\{1-(1-\alpha)\left[\psi+\left(\lambda_{h}+\kappa_{h} \gamma_{h}\right) A_{h}\right]\right\}, \\
x^{\star}= & \bar{l}(1-\alpha)\left(\psi+A_{h}\right), \\
c^{\star}= & \bar{l}\left\{1-(1-\alpha)\left[\psi+\left(\lambda_{h}+\kappa_{h} \gamma_{h}\right) A_{h}\right]\right\}^{\alpha}\left\{(1-\alpha)\left(\psi+A_{h}\right)\right\}^{1-\alpha} \\
& \text { with } A_{h}=\frac{1-\psi\left\{1-\alpha\left[1-\lambda_{h}-\kappa_{h}\left(\rho+\gamma_{h}\right) \exp \left[-\rho \sigma_{h}\right]\right]\right\}}{\lambda_{h}+\kappa_{h}\left\{(1-\alpha) \gamma_{h}+\alpha\left(\rho+\gamma_{h}\right) \exp \left[\rho \sigma_{h}\right]\right\}} .
\end{aligned}
$$

In addition, the stationary state is a saddle point.

Proof In the appendix.

Although only one of the two alternative energy producing techniques prevails in the stationary state in general, this is not necessarily true, if $\mathrm{EPT}_{1}$ and $\mathrm{EPT}_{2}$ have the same unit costs of energy, i.e., $\lambda_{1}+\kappa_{1}\left(\rho+\gamma_{1}\right) \exp \left[\rho \sigma_{1}\right]=\lambda_{2}+\kappa_{2}\left(\rho+\gamma_{2}\right) \exp \left[\rho \sigma_{2}\right]$. In this case, the society is indifferent in the optimal stationary state between $\mathrm{EPT}_{1}$ and $\mathrm{EPT}_{2}$, and the stationary state is no longer unique. As this only holds for very specific values of the exogenously given parameters, it is not further addressed in the following.

\section{Transition dynamics}

Although Proposition 2 shows that in the (medium-term) stationary state only one alternative energy producing technique prevails, this does not tell much about the transition path. In fact, it might be optimal to utilize both alternative energy producing techniques for some time along the optimal transition path. In the following, this is shown with a numerical example, as the transition paths cannot be analyzed analytically. Note that the parameter values used for the numerical optimization have been primarily chosen such, as to illustrate clearly the different effects, and, therefore, do not necessarily reflect the characteristics of real energy technology transitions. 
Figure 1 shows typical numerical optimization results for two scenarios. In the first scenario, $\mathrm{EPT}_{1}$ prevails in the stationary state (left side of Fig. 1), in the second scenario, $\mathrm{EPT}_{2}$ prevails in the stationary state (right side of Fig. 1). ${ }^{7}$ The exogenous parameters and the functional form of the instantaneous welfare function $V$ are given in Table 1. They are such that, without an emission constraint, 0.5 units of labor are employed in $\mathrm{EPT}_{0}$ to produce energy and 0.5 units of labor are directly employed in the consumption good process (8), to yield a constant consumption output of 0.5 units. Furthermore, given the exogenous parameters, energy saving alone under the binding emission constraint $\psi$ is optimal, if the unit costs of energy for both $\mathrm{EPT}_{1}$ and $\mathrm{EPT}_{2}$ exceed 3. To illustrate the effect of different dynamic characteristics, EPT 1 and $\mathrm{EPT}_{2}$ exhibit identical $\lambda_{h}$ and $\kappa_{h}(h=1,2)$ and differ only in $\sigma_{h}$ and $\gamma_{h}$. The investment lag for $\mathrm{EPT}_{2}$ is considered to be twice as long as for $\mathrm{EPT}_{1}$.

Note that the optimal paths exhibit oscillatory behavior, which is increasingly damped and finally converges toward the stationary state values. Although quite uncommon in simple capital accumulation models, oscillatory optimal paths are a general feature of timelagged optimal control problems, as shown in Appendix A.4. In the beginning, consumption drops drastically, as energy production via $\mathrm{EPT}_{0}$ is restricted by the emission constraint and a considerable amount of labor is bound to be invested in the alternative energy techniques. In the stationary state, consumption consolidates at a level of 0.488 , compared to 0.5 before the emission constraint became effective. Total energy output is below, and labor used in the consumption good process (8) is higher than in the original stationary state.

The most notable characteristic of these scenarios, however, is that it can be optimal to utilize both alternative techniques along the optimal path. In the case that $\mathrm{EPT}_{1}$ prevails in the stationary state, the deterioration rates have been set to $\gamma_{1}=0.1$ and $\gamma_{2}=0.15$, implying unit costs of energy equal to 1.158 for $\mathrm{EPT}_{1}$, and 1.221 for $\mathrm{EPT}_{2}$. Hence, as both unit costs of energy are below the threshold 3, where energy saving alone would be optimal, investment in both alternative energy producing techniques cannot be ruled out by Proposition 1 . However, as $\mathrm{EPT}_{1}$, the energy producing technique with the smaller investment lag, prevails in the stationary state, $\mathrm{EPT}_{2}$ is never utilized over the whole time horizon.

The situation is different in the second scenario, where $\mathrm{EPT}_{2}$ prevails in the stationary state. Here, the deterioration rates have been set to $\gamma_{1}=0.15$ and $\gamma_{2}=0.1$, implying unit costs of energy equal to 1.210 for $\mathrm{EPT}_{1}$, and 1.166 for $\mathrm{EPT}_{2}$. Although $\mathrm{EPT}_{2}$ prevails in the stationary state, $\mathrm{EPT}_{1}$ is utilized in the beginning. This asymmetry is quite intuitive: although $\mathrm{EPT}_{2}$ exhibits lower unit costs in the stationary state, $\mathrm{EPT}_{1}$ has the advantage of a shorter investment lag. Thus, in the short run, EPT 1 can (at least partly) bridge the gap between the investment and the emergence of capital for $\mathrm{EPT}_{2}$. Obviously, this is not possible the other way round. When $\mathrm{EPT}_{1}$ exhibits lower unit costs in the stationary state, it is also advantageous in the short run, due to its shorter investment lag. However, this is not necessarily true for other economic contexts. In the second scenario, $\mathrm{EPT}_{2}$ exhibits a smaller deterioration rate and, thus, its capital stock lasts longer. If, for example, a finite instead of an infinite time horizon is considered, this dynamic characteristic of higher durability would make $\mathrm{EPT}_{2}$ preferable over $\mathrm{EPT}_{1}$ at the end of the time horizon.

\footnotetext{
7 The numerical optimizations were derived with the advanced optimal control software package MUSCODII (Diehl et al. 2001), which exploits the multiple shooting state discretization (Leineweber et al. 2003). To apply MUSCOD-II to time-lagged optimal control problems, the optimization problem has to be reformulated as described in Winkler et al. (2004).
} 
$E P T_{1}$ prevails in the long-run

Consumption production

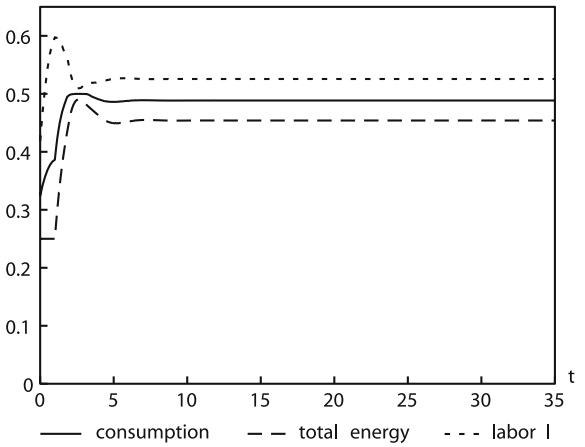

EPT $_{1}$

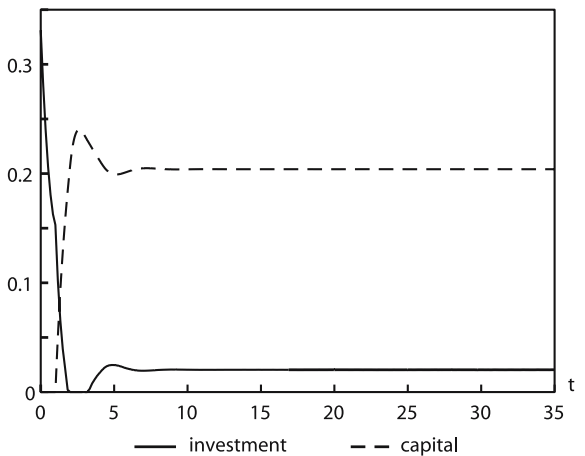

$\mathrm{EPT}_{2}$

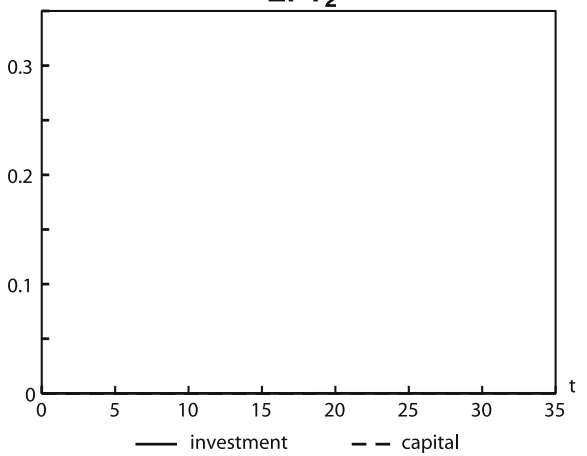

$E P T_{2}$ prevails in the long-run

Consumption production

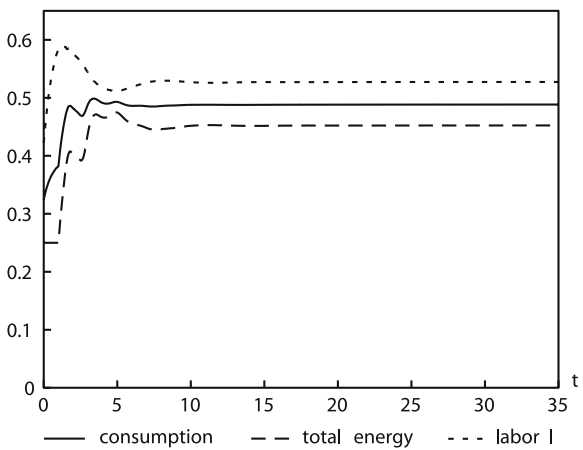

$\mathrm{EPT}_{1}$

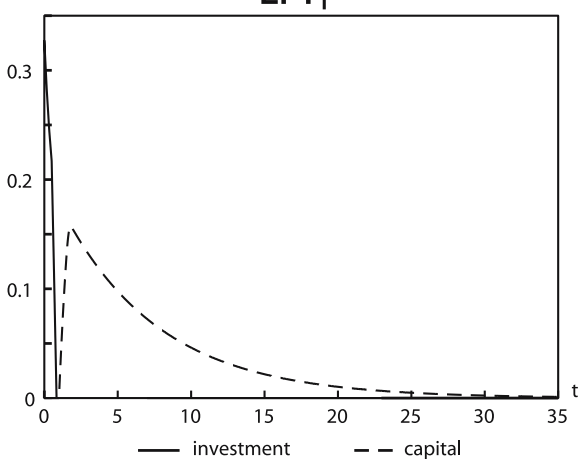

$\mathrm{EPT}_{2}$

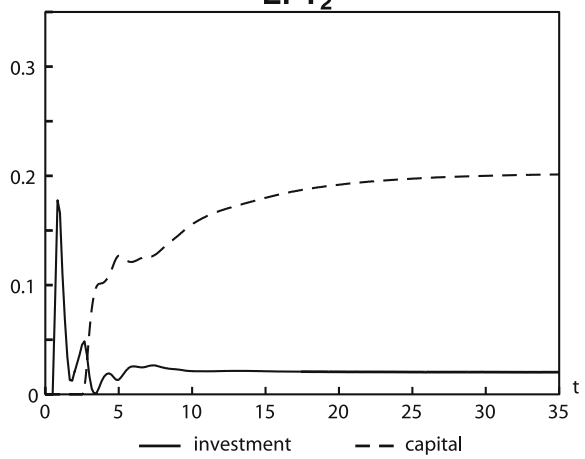

Fig. 1 Two typical optimal paths for the case that $\mathrm{EPT}_{1}$ (left) and $\mathrm{EPT}_{2}$ (right) prevails in the stationary state. Optimal paths are shown for consumption, total energy supply $x$ and labor $l$ employed in the consumption good process (top), investment and capital stock of EPT 1 (middle), and investment and capital stock of EPT 2 (bottom)

Table 1 Exogenous parameter values for the numerical optimization

\begin{tabular}{lllllllllll}
\hline$\rho$ & $\alpha$ & $\psi$ & $\kappa_{1}$ & $\kappa_{2}$ & $\lambda_{1}$ & $\lambda_{2}$ & $\sigma_{1}$ & $\sigma_{2}$ & $\bar{l}$ & $V(c(t))$ \\
\hline 0.05 & 0.5 & 0.5 & 1 & 1 & 1 & 1 & 1 & 2 & 1 & $\ln (c(t))$ \\
\hline
\end{tabular}




\section{Discussion}

The main result of the model set up in this paper is that the choice of technique, while trivial in the short-run point of view of a static economy, and mainly determined by technological change in the long run, gains particular importance in the medium-term perspective, where the time horizon is too short for technological change to occur, but long enough for specific capital stocks to build up and to deteriorate. It is shown that along the optimal path, and depending on the dynamic characteristics, production technologies are used during the transition period, although they are inferior in the stationary state. In the following, I briefly discuss the robustness of this result in accordance to the model assumptions and hint at resulting policy implications.

\subsection{Model assumptions}

The driving denominators for the result are the dynamic characteristics. For the sake of simplicity, I only consider two straight forward dynamic characteristics in the model, the time-lag $\sigma$ and the deterioration rate $\gamma$. They determine the two time scales on which capital builds up and declines, respectively. While the deterioration rate is a standard assumption in models including capital, the investment lag is rather a specialty. However, its consideration is straightforward, as energy plant construction exhibits both considerably long and strongly varying construction periods, depending on the type of the plant. According to IEA (2005), the construction time for gas fired plants range between 2 and 3 years, most coal fired plants are set up within 4 years and nuclear power plants need 5-10 years to build. Most renewable power generation plants are of considerably smaller size and exhibit construction periods of less than 2 years. Exceptions are macro hydroelectric power plants such as the Three Gorges Dam Project in China, which has a scheduled construction period of 17 years. Also the costs per kWh vary drastically among the different energy producing techniques. According to the same study (IEA 2005), the costs per kWh range between 2.5 and 6 USD cent for coal fired plants, between 3.7 and 6.3 USD cent for gas fired plants and between 2.1 and 5 USD cent for nuclear power plants. Among the renewable energies, wind energy exhibits costs per kWh between 3.5 and 14 USD cent and micro-scale hydro power plants range between 4 and 10 USD cent per $\mathrm{kWh}$. Solar power is most expensive implying costs per $\mathrm{kWh}$ ranging between at least 15 USD cent up to considerably beyond 30 USD cent.

The linear-limitational form of the energy technologies is a crucial assumption for the results. The linearity assures that production techniques might not be used at all, as their unit costs (in terms of labor), which are independent of the output level, are too high. Obviously, this could not happen, if all technologies satisfy Inada conditions and, thus, would always be utilized (although, possibly on very low levels). As already pointed out in Sect. 2, the linear-limitational form accounts better for the technical and thermodynamical rigidities in energy production compared to a Cobb-Douglas or CES function.

Note, however, that it may be optimal to utilize both techniques does not depend on an unanticipated emission constraint. Even if society knows in advance that a binding emission target will be enacted in the future, the qualitative result that both alternative techniques may be utilized along the optimal transition path, is robust as shown in Winkler (2003, Chap. 7). The intuition is that, even if society knows about the emission constraint in advance, it is suboptimal to start investing in the alternative techniques too early, as they are inefficient compared to the status quo technique, as long as the emission constraint is not enacted. In summary, the model result does not (at least qualitatively) depend on the specific assumption of a binding emission target, which is enacted without lead-in time. Moreover, as already 
pointed out in the introduction, the case of an emission target is of particular relevance in the medium-term perspective of climate change mitigation.

From a more technical perspective, the time-to-build feature is responsible for the oscillatory behavior of the optimal paths (see Fig. 1). It is worth noting that this is a general characteristic of functional differential equations and is not an artifact of specific model assumptions such as the liner-limitational form of the production functions or a binding and unanticipated emission constraint. ${ }^{8}$ However, both the amplitude and the persistence of these oscillations depend on the explicit model setup and the values of the exogenous parameters. ${ }^{9}$ Thus, one has to be cautious about the empirical relevance of the oscillatory behavior. In particular, this holds as the simple model used in this paper allows for costless shift of labor between the different sectors, which is a very optimistic assumption about labor mobility.

For the sake of a tractable model, I abstract from a series of peculiarities of the economics of the energy industry. First, energy technologies differ in more dynamic characteristics than just the construction period and the deterioration rate. As an example, think of different turn-on/turn-off costs and rigidities, which play an important role for the choice (or the mix) of techniques, as the energy industry exhibits oscillatory demand fluctuations on different time scales, such as day/night time and summer/winter (e.g., hydroelectric power and gas cogeneration plants can easily be turned on and switched off and are, therefore, well suited to supply energy for peak demands, while nuclear power plants are extremely costly and time-consuming to load adjustments and, thus, are preferably used to supply the base load).

Second, the energy industry exhibits an oligopolistic market structure. As is well known from the industrial organization literature, unregulated oligopolistic market regimes lead in general to market failures in the sense that the market outcome is not a social optimum. Thus, the social optimum, as derived in this model, cannot simply be implemented by a decentralized unregulated market regime. In this paper, I abstract from these market failures to concentrate on the optimal choice of technique.

\subsection{Policy implications}

Although the results stem from a highly stylized theoretical model, there are some direct and indirect policy conclusions to be drawn. First, the direct policy implication, concerning optimal compliance with emission constraints, is straightforward. Although one technique has the smallest unit costs of energy and, thus, is the prime candidate to replace the established and polluting technique, it might be beneficial to look also at energy techniques with higher unit costs of energy, as they might have favorable dynamic characteristics, which can help to smoothen the transition path. Hence, the explicit consideration of the different dynamic characteristic offers additional cost-saving potential for the transition toward a less polluting energy industry in the medium-term perspective.

Second, in light of the model results, policies which subsidize clean energy technologies, such as the German "Erneuerbare-Energien-Gesetz" (Renewable Energy Act), have to be reconsidered according to their economic efficiency. The Renewable Energy Act was first enacted in April 2000 and amended in August 2004. The stated purpose of the act is to increase the share of renewable energy technologies to at least $12.5 \%$ in 2020 and $20 \%$ in 2020. Moreover, the act aims at a sustainable provision of energy, a reduction of insecure markets for fossil fuels and a promotion of technological progress in renewable energy

\footnotetext{
8 See, for example, Winkler (2004) for a model with non-linear production function and explicit economic valuation of the trade-off between utility derived from consumption versus the disutility stemming from stock pollution.

9 Winkler et al. (2005) investigate the dependence between oscillatory behavior and the size of the time-lag.
} 
technology. ${ }^{10}$ Thus, the renewable energy act plays an important role in the endeavor to comply with the $\mathrm{CO}_{2}$ emissions reduction target of $25 \%$. On the one hand, the model results give additional support for the act, as renewable energy technologies exhibit, in general, higher unit costs but smaller construction periods than fossil fuel based or nuclear power plants and, thus, are potential candidates for smoothing transition paths in the medium-term perspective. On the other hand, if unit costs are above a certain threshold, energy saving is the cost-efficient method to comply. In fact, at least from a medium-term perspective, which does not take into account dynamic effects like learning curves or induced technological change, the very high subsidies for photovoltaics (45.7-62.4 EUR cent per $\mathrm{kWh}$ compared to 5.5-9.1 EUR cent per kWh for wind energy and 7.16-15 EUR cent per kWh for geothermal energy) raise doubts about their efficient use in Germany.

Third, the results are not restricted to the special context of emission constraints in the context of climate change mitigation. Every kind of institutional change creates a shock, which alters the economic context. Thus, established techniques may be less favorable afterwards and techniques, which were inefficient before, may now be potential candidates to replace the established techniques, either because they are now the most efficient technique (i.e., the technique with the lowest unit costs) or because they have favorable dynamic characteristics for smoothing the transition path. Thus, the medium-term perspective introduced in this paper, which is too short for technological change to occur, but long enough for capital stocks to build up and deteriorate, might provide a useful framework to identify additional cost-saving potential for a variety of different contexts.

\section{Conclusions}

In this paper, I have analyzed, how an economy optimally complies to a binding emission constraint that restricts the use of a established energy technique. I have shown that energy saving alone is optimal only if both alternative techniques exhibit unit costs of energy over a certain threshold. Otherwise, the alternative energy technique with the lower unit costs of energy will prevail in the stationary state. On the transition path, however, it may be beneficial for the society to utilize both techniques.

These results imply that the medium-term perspective taken in this paper may offer additional cost-saving potential by utilizing techniques during the transition period, although they are not optimal in the (medium-term) stationary state. In the context of binding emission constraints to mitigate anthropogenic climate change, this implies that the unit costs of energy should not be the prime determinant in the political discussion for the choice of technique, but other dynamic characteristics, such as the construction period, the lifetime, etc., have to be taken into account. However, the model presented here does only consider the medium-term perspective. For a successful mitigation policy, all three time scales have to be taken into account. In particular, this implies an endogenous emission target and explicit consideration of learning curves and induced technological change.

Nevertheless, the first (international) agreements on $\mathrm{CO}_{2}$ emission reductions are enacted, and the compliance with these targets is a task that many industrialized countries face today. The results of the model constitute a theoretical underpinning to evaluate the economic efficiency of policies, which aim to reduce emissions in order to comply with a specific reduction target, such as the German Renewable Energy Act. Even if some renewable energy

10 According to the act, suppliers of renewable energy get unit subsidies which depend on the energy technology and the size of the plant. Moreover, the subsidies are decreasing over time, to give incentives for productivity increases. The subsidy rates are adjusted regularly (last adjustment in August 2004). 
technologies might not be the optimal choice in the long run, they might still be favorable along the transition path. However, if and to what extend subsidies for different energy technologies are 'economically justified' has to be scrutinized by further empirical research, which also takes into account the specific peculiarities of the energy industry.

Although this paper was explicitly framed to analyze the compliance with binding emission targets in the context of mitigating climate change, the results point to broader implications. So far I have only considered a foreseeable shock as a result of a deliberate policy action. However, we live in an unpredictable world. My results suggest that we are better prepared for unforeseeable shocks the more different techniques, with all kinds of different dynamic characteristics, we have available. The situation is comparable to agriculture, where it is well known that monocultures might yield the highest harvest, but crop combinations reduce the systems sensitivity to exogenous shocks, such as drought, flooding or epidemics. Hence, a bundle of techniques, with a high diversity of dynamic characteristics, can act as an insurance against unforeseeable exogenous shocks, a notion briefly mentioned in Baumgärtner and Schiller (2001). This constitutes another fruitful direction for future research.

\section{Appendix}

\section{A.1 Proof of condition (19)}

In the status quo stationary state, the labor restriction reduces to $l=\bar{l}-l_{0}$ and total energy production equals $x=x_{0}=l_{0}$. Utilizing condition (16b) that $F_{l}=F_{x}$, if the emission target is not binding, leads to the following values for the stationary state without binding emission target:

$$
\begin{aligned}
& l^{\star}=\alpha \bar{l}, \\
& l_{0}^{\star}=x^{\star}=e^{\star}=(1-\alpha) \bar{l}, \\
& c^{\star}=\alpha^{\alpha}(1-\alpha)^{1-\alpha} \bar{l} .
\end{aligned}
$$

Thus, to be binding, $\phi<e^{\star}$.

\section{A.2 Proof of Condition (21)}

Assume that the emission target (3) is not binding and that the utilization of $\mathrm{EPT}_{0}$ alone is optimal. Then the economy stays in the status quo stationary state given by (A.1). Utilizing that $F_{x}=F_{l}$, if the emission target is not binding, one obtains for the shadow prices of capital (17):

$$
p_{k_{h}}(t)=\frac{1-\lambda}{\kappa_{h}\left(\rho+\gamma_{h}\right)} V_{c}\left(c^{\star}\right) F_{l}\left(l^{\star}, x^{\star}\right) \exp [-\rho t], \quad h=1,2 .
$$

Furthermore, $i_{h}=0$ and, according to conditions (16e), $p_{i_{h}} \geq 0(h=1,2)$. Then, inserting (16a) and (A.2) in conditions (16c) yields:

$$
V_{c}\left(c^{\star}\right) F_{l}\left(l^{\star}, x^{\star}\right) \exp [-\rho t] \geq \frac{1-\lambda}{\kappa_{h}\left(\rho+\gamma_{h}\right)} V_{c}\left(c^{\star}\right) F_{l}\left(l^{\star}, x^{\star}\right) \exp \left[-\rho\left(t+\sigma_{h}\right)\right], \quad h=1,2 .
$$

Note that this condition is invariant over time, i.e., is the same for all $t$. This is true, as in a stationary state both the welfare loss due to a marginal investment in capital and the welfare 
gain due to the future utilization of the capital good are constant over time. Dividing both sides by $V_{c}\left(c^{\star}\right) F_{l}\left(l^{\star}, x^{\star}\right) \exp [-\rho t]$ and re-arranging the inequality leads to the conditions (21).

\section{A.3 Proof of Proposition 1}

Consider a binding emission target (20) and that it is not optimal to invest in either of the two alternative energy producing technologies for all $t$. Then, the labor restriction reduces to $l(t)=\bar{l}-l_{0}(t)$. Furthermore, the usage of $\mathrm{EPT}_{0}$ is determined by the emission target and, thus, the economy stays in the following stationary state:

$$
\begin{aligned}
\hat{l} & =[1-\psi(1-\alpha)] \bar{l}, \\
\hat{l}_{0} & =\hat{x}=\hat{e}=\psi(1-\alpha) \bar{l}, \\
\hat{c} & =[1-\psi(1-\alpha)]^{\alpha}[\psi(1-\alpha)]^{1-\alpha} \bar{l} .
\end{aligned}
$$

Following the same line of argument as in appendix A.2, this is only optimal if:

$$
V_{c}(\hat{c}) F_{l}(\hat{l}, \hat{x}) \exp [-\rho t] \geq \frac{F_{x}(\hat{l}, \hat{x})-\lambda_{h} F_{l}(\hat{l}, \hat{x})}{\kappa_{h}\left(\rho+\gamma_{h}\right)} V_{c}(\hat{c}) \exp \left[-\rho\left(t+\sigma_{h}\right)\right], \quad h=1,2 .
$$

Dividing both sides by $V_{c}(\hat{c}) F_{l}(\hat{l}, \hat{x}) \exp [-\rho t]$, inserting $\hat{l}$ and $\hat{x}$ from (A.4) and re-arranging the inequality, leads to the conditions (22).

\section{A.4 Proof of proposition 2}

In the stationary state equations (16c) yield after inserting Eq. (16a):

$$
V_{c}\left(c^{\star}\right) F_{l}\left(l^{\star}, x^{\star}\right) \exp [-\rho t]=p_{k_{1}}\left(t+\sigma_{1}\right)+p_{i_{1}}(t)=p_{k_{2}}\left(t+\sigma_{2}\right)+p_{i_{2}}(t) .
$$

Solving Eq. (17) for the stationary state, and inserting in Eq. (A.6), one obtains:

$$
F_{l}^{\star}=\frac{F_{x}\left(l^{\star}, x^{\star}\right)-\lambda_{1} F_{x}\left(l^{\star}, x^{\star}\right)}{\kappa_{1}\left(\rho+\gamma_{1}\right) \exp \left[\rho \sigma_{1}\right]}+p_{i_{1}}(t)=\frac{F_{x}\left(l^{\star}, x^{\star}\right)-\lambda_{2} F_{x}\left(l^{\star}, x^{\star}\right)}{\kappa_{2}\left(\rho+\gamma_{2}\right) \exp \left[\rho \sigma_{2}\right]}+p_{i_{2}}(t) .
$$

Assuming that either $p_{i_{1}}(t)=0$ or $p_{i_{2}}(t)=0$ (which has to be the case, as conditions (22) are violated and, thus, energy saving alone is not optimal) one can show that:

$$
\begin{aligned}
p_{i_{h}}(t)>0 \Leftrightarrow & \lambda_{h}+\kappa_{h}\left(\rho+\gamma_{h}\right) \exp \left[\rho \sigma_{h}\right]>\lambda_{j}+\kappa_{j}\left(\rho+\gamma_{j}\right) \exp \left[\rho \sigma_{j}\right], \\
& h, j=1,2, \quad h \neq j .
\end{aligned}
$$

Thus, in the optimal stationary state, only the alternative energy producing technique is utilized, which has the lower unit costs of energy.

In order to obtain the stationary state values for the control and stock variables, assume that $\mathrm{EPT}_{h}$ prevails, i.e., $p_{i_{h}}(t)=0$. Then, Eq. (A.6) together with the equation of motion (14c) build a system of two (implicit) equations for the control variable $i_{h}$ and the stock variable $k_{h}$ :

$$
\begin{aligned}
\frac{F_{x}\left(l^{\star}, x^{\star}\right)}{F_{l}\left(l^{\star}, x^{\star}\right)} & =\lambda_{h}+\kappa_{h}\left(\rho+\gamma_{h}\right) \exp \left[\rho \sigma_{h}\right], \\
k_{h}^{\star} & =\gamma_{h} i_{h}^{\star} .
\end{aligned}
$$

Solving these equations yields (23).

To determine the stability properties of the stationary state, I analyze the system dynamics of the optimization problem (14). As there is only investment in one of the two alternative 
energy producing techniques in the stationary state, the optimization problem (14) reduces to a optimal control problem with only one control and one stock variable in a neighborhood around the stationary state (as $l_{0}$ is completely determined by the emission target and either $i_{1}=k_{1} \equiv 0$ or $i_{2}=k_{2} \equiv 0$, depending on which of the two alternative energy producing techniques has the lower unit costs of energy). Assuming that $\mathrm{EPT}_{h}$ prevails in the stationary state, the system dynamics in a neighborhood around the stationary state is fully represented by the following system of differential equations, which can be derived by the necessary and sufficient conditions: ${ }^{11}$

$$
\begin{gathered}
\dot{i}_{h}(t)=\frac{V_{c}\left(t+\sigma_{h}\right)\left[F_{x}\left(t+\sigma_{h}\right)-\lambda_{h} F_{l}\left(t+\sigma_{h}\right)\right] \exp [-\rho \sigma]-V_{c}(t) F_{l}(t) \kappa_{h}(\gamma+\rho)}{\kappa_{h}\left[V_{c c}(t) F_{l}(t)^{2}+V_{c}(t) F_{l l}(t)\right]} \\
+\frac{V_{c c}(t) F_{l}(t)\left[\lambda_{h} F_{l}(t)-F_{x}(t)\right]+V_{c}(t)\left[\lambda_{h} F_{l l}(t)-F_{l x}(t)\right]}{\kappa_{h}\left[V_{c c}(t) F_{l}(t)^{2}+V_{c}(t) F_{l l}(t)\right]}\left[\gamma_{h} k_{h}(t)-i_{h}\left(t-\sigma_{h}\right)\right], \\
\dot{k}_{h}(t)=i_{h}\left(t-\sigma_{h}\right)-\gamma_{h} k_{h}(t) .
\end{gathered}
$$

As $\dot{i}$ and $\dot{k}$ also depend on advanced (i.e., at a later time) and on retarded (i.e., at an earlier time) variables, Eqs. (A.11) and (A.12) form a system of functional differential equations. ${ }^{12}$ Although this system is not analytically soluble in general (not even in the linear approximation around the stationary state), the stationary state (23) is in general saddle point stable. ${ }^{13}$ To see this, I introduce the following abbreviations (for the sake of a more convenient presentation, I drop the subscript $h$ ):

$$
\begin{aligned}
V_{i}(t) & =-V_{c}(t) F_{l}(t), \\
V_{k}(t) & =V_{c}(t)\left[\frac{1}{\kappa} F_{x}(t)-\frac{\lambda}{\kappa} F_{l}(t)\right], \\
V_{i i}(t) & =V_{c c}(t) F_{l}(t)^{2}+V_{c}(t) F_{l l}(t), \\
V_{k k}(t) & =V_{c c}(t)\left[\frac{1}{\kappa} F_{x}(t)-\frac{\lambda}{\kappa} F_{l}(t)\right]+V_{c}(t)\left[\frac{1}{\kappa^{2}} F_{x x}(t)-\frac{2 \lambda}{\kappa^{2}} F_{x l}(t)+\frac{\lambda^{2}}{\kappa^{2}} F_{l l}(t)\right], \\
V_{i k}(t) & =V_{c c}(t) F_{l}(t)\left[\frac{\lambda}{\kappa} F_{l}(t)-\frac{1}{\kappa_{h}} F_{x}(t)\right]+V_{c}(t)\left[\frac{\lambda}{\kappa} F_{l l}(t)-\frac{1}{\kappa} F_{l x}(t)\right] .
\end{aligned}
$$

Thus, one obtains for the functional differential equation system (A.11)-(A.12):

$$
\begin{aligned}
& \dot{i}(t)=\frac{V_{i}(t)}{V_{i i}(t)}(\gamma+\rho)+\frac{V_{k}(t+\sigma)}{V_{i i}(t)} \exp [-\rho \sigma]+\frac{V_{i k}(t)}{V_{i i}(t)}(\gamma k(t)-i(t-\sigma)), \\
& \dot{k}(t)=i(t-\sigma)-\gamma k(t) .
\end{aligned}
$$

11 Differentiate Eq. (16c) with respect to $t$, insert in Eq. (16d) and solve for $\dot{i}_{h}$.

12 For an introduction to functional differential equations see Asea and Zak (1999, Sect. 2) and Gandolfo (1996, Chap. 27). A detailed exposition for linear functional differential equations is given in Bellman and Cooke (1963), and Hale (1977).

13 The system of functional differential Eqs. (A.11)-(A.12) may also exhibit so called limit-cycles, i.e., the optimal paths oscillate around the stationary state without converging toward or diverging from it (e.g., Feichtinger et al. 1994; Asea and Zak 1999; Liski et al. 2001 and Wirl 1995,1999,2002). 
In the linear approximation around the stationary state (23), the differential equation system (A.13) yields:

$$
\begin{aligned}
\dot{i}(t) \approx & (\gamma+\rho)\left(i(t)-i^{\star}\right)+\frac{V_{i k}}{V_{i i}} \exp [-\rho \sigma]\left(i(t+\sigma)-i^{\star}\right)-\frac{V_{i k}}{V_{i i}}\left(i(t-\sigma)-i^{\star}\right) \\
& +\frac{V_{i k}}{V_{i i}}(2 \gamma+\rho)\left(k(t)-k^{\star}\right)+\frac{V_{k k}}{V_{i i}} \exp [-\rho \sigma]\left(k(t+\sigma)-k^{\star}\right)+i^{\star}, \\
\dot{k}(t) \approx & \left(i(t-\sigma)-i^{\star}\right)-\gamma\left(k(t)-k^{\star}\right)+k^{\star} .
\end{aligned}
$$

Linear functional differential equations are also called differential-difference equations. Similar to the case of ordinary linear first-order differential equations, the elementary solutions for $i_{h}$ and $k_{h}$ are exponential functions, and the general solution is given by the superposition of the elementary solutions

$$
i(t)=i^{\star}+\sum_{n} i_{n} \exp \left[x_{n} t\right], \quad k(t)=k^{\star}+\sum_{n} k_{n} \exp \left[x_{n} t\right]
$$

where the $i_{n}$ and $k_{n}$ denote constants which can (at least in principle) be unambiguously determined by the set of initial conditions and the transversality condition. The eigenvalues $x_{n}$ are the roots of the characteristic polynomial $Q(x)$. The characteristic polynomial $Q(x)$ for the system of differential-difference equations (A.14) is given by the determinant of the Jacobian of (A.14) minus the identity matrix times $x$ :

$$
\begin{aligned}
Q(x)= & x^{2}-x\left[\rho+\frac{V_{i k}}{V_{i i}}(\exp [\sigma(x-\rho)]-\exp [-\sigma x])\right] \\
& -\frac{V_{i k}}{V_{i i}} \exp [-\sigma x](\rho+2 \gamma)-\frac{V_{k k}}{V_{i i}} \exp [-\rho \sigma]-\gamma(\gamma+\rho) .
\end{aligned}
$$

$Q(x)$ is a quasi-polynomial, which exhibits an infinite number of complex roots. To determine whether the stationary state is a saddle point, one needs to determine the signs of the real parts of the characteristic roots. In fact, the characteristic polynomial $Q(x)$ has an infinite number of roots with negative real part and an infinite number of roots with positive real part and, thus, the stationary state is a saddle point.

To see this, note first that the characteristic roots are symmetric around $\rho / 2$, i.e., if $x^{0}$ is a characteristic root, then $\rho-x^{0}$ is also a characteristic root (one can easily verify that $\left.Q\left(x^{0}\right)=Q\left(\rho-x^{0}\right)\right)$. Second, we introduce the new variable $y=\sigma x$ and multiply $Q$ with $\sigma^{2} \exp [y]$

$$
\begin{aligned}
Q(y)= & y^{2} \exp [y]-\sigma y\left[\rho \exp [y]+\frac{V_{i k}}{V_{i i}}(\exp [2 y-\rho \sigma]-1)\right] \\
& -\sigma^{2}\left[\frac{V_{i k}}{V_{i i}}(2 \gamma+\rho)-\frac{V_{k k}}{V_{i i}} \exp [y-\rho \sigma]-\gamma(\gamma+\rho) \exp [y]\right],
\end{aligned}
$$

in order to apply Theorem 13.1 of Bellman and Cooke (1963, p. 441). As $Q(y)$ has no principal term, i.e., a term where the highest power of $y$ and the highest exponential term appear jointly, ${ }^{14} Q(y)$ has "an unbounded number of zeros with arbitrarily large positive real part" (ibid). But as the characteristic roots are symmetric around $\rho / 2$, this also implies an unbounded number of roots with arbitrarily large negative real part.

14 In this case, the principal term would be a term with $y^{2} \exp [2 y]$. 
Acknowledgements I am grateful to Stefan Baumgärtner, Malte Faber, Jakub Kronenberg, John Proops, Edwin van der Werf and two anonymous reviewers for valuable comments on an earlier draft, and to the Simulation and Optimization Group of the Interdisciplinary Center for Scientific Computing, University of Heidelberg, for a free license of the MUSCOD-II software package. Financial support by the Deutsche Forschungsgemeinschaft (German Research Foundation) under the graduate programme "Environmental and Resource Economics" and by the European Commission under the Marie Curie Intra-European Fellowship scheme, No. MEIF-CT-2003-501536, is gratefully acknowledged.

\section{References}

Aaheim H (1999) Climate policy with multiple sources and sinks of greenhouse gases. Environ Resour Econ $14: 413-429$

Aidt TS, Dutta J (2004) Transitional politics: emerging incentive-based instruments in environmental regulation. J Environ Econ Manage 47:458-479

Asea PK, Zak PJ (1999) Time-to-build and cycles. J Econ Dyn Control 23:1155-1175

Baumgärtner S, Schiller J (2001) Vielfalt und Nachhaltigkeit. Der Einfluss von Beständen und des Zeithorizonts auf zukünftige ökonomische Wahlmöglichkeiten. Z Angew. Umweltforschung, Special issue 13/2001:137-148

Bellman R, Cooke KL (1963) Differential-difference equations. Academic, New York

Bhaduri A (1968) An aspect of project selection: durability vs. construction-period. Econ J 78:344-348

von Böhm-Bawerk E([1889]1921) Kapital und Kapitalzins. Positive Theorie des Kapitals (Capital and Interest. The Positive Theory of Capital), 4th edn. First published in 1889. Macmillan, London

Böhringer C (2003) The Kyoto protocol: a review and perspectives. Oxford Rev Econ Policy 19:451-466

Böhringer C, Vogt C (2003) Economic and environmental impacts of the Kyoto protocol. Can J Econ 36:475494

Boucekkine R, Licandro O, Puch LA, del Rio F (2005) Vintage capital and the dynamics of the AK model. J Econ Theory 120:39-72

Buonanno P, Carraro C, Galeotti M (2003) Endogenous induced technical change and the costs of Kyoto. Resour Energy Econ 25:11-34

Carraro C, Gerlagh R, van der Zwaan B (2003) Endogenous technical change in environmental maroeconomics. Resour Energy Econ 25:1-10

Chichilnisky G (1996) An axiomatic approach to sustainable development. Soc Choice Welf 13:231-257

Dellink R, Hofkes M, van Ierland E, Verbruggen H (2004) Dynamic modelling of pollution abatement in a CGE framework. Econ Model 21:965-989

Diehl M, Leineweber DB, Schäfer AAS (2001) Muscod-II users' manual. Preprint 2001-25, Interdisciplinary Center for Scientific Computing, University of Heidelberg, Heidelberg

El-Hodiri MA, Loehman E, Whinston A (1972) An optimal growth model with time lags. Econometrica 40:1137-1146

Endres A, Finus M (1999) International environmental agreements: how the policy instrument affects equilibrium emissions. J Inst Theor Econ 155:527-550

Endres A, Finus M (2002) Quotas may beat taxes in a global emission game. Int Tax Public Finance 9:687-707

Faber M (1979) Introduction to modern Austrian capital theory. Springer, Heidelberg

Falk I, Mendelsohn R (1993) The economics of controlling stock pollutants: an efficient strategy for greenhouse gases. J Environ Econ Manage 25:76-88

Feichtinger G, Hartl RF, Kort PM, Veliov MV (2006) Anticipation effects of technological progress on capital accumulation: A vintage capital approach. J Econ Theory 126:143-164

Feichtinger G, Novak A, Wirl F (1994) Limit cycles in intertemporal adjustment models: theory and application. J Econ Dyn Control 18:353-380

Fisher C, Parry I, Pizer W (2003) Instrument choice for environmental protection when technological innovation is endogenous. J Environ Econ Manage 45:523-545

Gandolfo G (1996) Economic dynamics, Third, completely revised and enlarged edition. Springer, Berlin

Gerlagh R, Lise W (2005) Carbon taxes: a drop in the ocean, or a drop that erodes the stone? The effect of carbon taxes on technological change. Ecol Econ 54:241-260

Gerlagh R, van der Zwaan B (2003) Gross world product and consumption in a global warming model with endogenous technological change. Resour Energy Econ 25:35-57

Gersbach H, Glazer A (1999) Markets and regulatory hold-up problems. J Environ Econ Manage 37:151-164

Gersbach H, Requate T (2004) Emission taxes and optimal refunding schemes. J Public Econ 88:713-725 
Gollier C, Treich N (2003) Decision-making under scientific uncertainty: the economics of the Precautionary Principle. J Risk Uncertain 27:77-103

Goulder LH, Mathai K (2000) Optimal $\mathrm{CO}_{2}$ abatement in the presence of induced technological change. J Environ Econ Manage 39:1-38

Goulder LH, Schneider SH (1999) Induced technological change and the attractiveness of $\mathrm{CO}_{2}$ abatement policies. Resour Energy Econ 21:211-253

Hale J (1977) Theory of functional differential equations. Springer, New York

Hicks JR (1973) Capital and time: a neo-Austrian theory. Clarendon, Oxford

Hoel M, Karp L (2000) Taxes versus quotas for a stock pollutant. Resour Energy Econ 24:367-384

IEA (2005) Projected costs of generating electricity—2005 update. OECD Nuclear Energy Agency (NEA) and International Energy Agency (IEA), Paris

Ioannides YM, Taub B (1992) On dynamics with time-to-build investment and non-separable leisure. J Econ Dyn Control 16:225-241

Karp L (2005) Global warming and hyperbolic discounting. J Public Econ 89:261-282

Kolstad CD (1996) Learning and stock effects in environmental regulation: the case of greenhouse gas emissions. J Environ Econ Manage 31:1-18

Kydland FE, Prescott EC (1982) Time to build and aggregate fluctuations. Econometrica 50:1345-1370

Laffont JJ, Tirole J (1996) Pollution permits and compliance strategies. J Public Econ 62:85-125

Lange A (2003) Climate change and the irreversibility effect-combining expected utility and maximin. Environ Resour Econ 25:417-434

Leineweber DB, Bauer I, Bock HG, Schlöder JP (2003) An efficient multiple shooting based reduced SQP strategy for large-scale dynamic process optimization-part I: theoretical aspects. Comput Chem Eng 27:157-166

Li CZ, Löfgren KG (2000) Renewable resources and economic sustainability: a dynamic analysis with heterogenous time preferences. J Environ Econ Manage 40:236-250

Lind RC (ed) (1982) Discounting for time and risk in energy policy. John Hopkins University Press, Baltimore

Liski M, Kort PM, Novak A (2001) Increasing returns and cycles in fishing. Resour Energy Econ 23:241-258

Löschel A (2002) Technological change in economic models of environmental policy: a survey. Ecol Econ 43:105-126

Moledina AA, Coggins JS, Polasky S, Costello C (2003) Dynamic environmental policy with strategic firms: prices versus quantities. J Environ Econ Manage 45:356-376

Montero JP (2002) Permits, standards, and technology innovation. J Environ Econ Manage 44:23-44

Moslener U, Requate T (forthcoming) Optimal abatement in dynamic multi-pollutant problems when pollutants can be complements or substitutes. J Econ Dyn Control

Newell RG, Jaffe AB, Stavins RN (1999) The induced innovation hypothesis and energy-saving technological change. Q J Econ 114:941-975

Newell RG, Pizer WA (2003) Regulating stock externalities under uncertainty. J Environ Econ Manage 45:416-432

Nordhaus WD (2002) Modeling induced innovation in climate-change policy. In Grübler A, Nakicenovic N, Nordhaus WD (eds) Technological change and the environment. Resources for the Future, pp 182-209

Nuti DM (1970) Capitalism, socialism and steady growth. Econ J 80:32-57

Pezzey JCV (2003) Emission taxes and tradeable permits: a comparison of views on long-run efficiency. Environ Resour Econ 26:329-342

Phaneuf DJ, Requate T (2002) Incentives for investment in advanced pollution abatement technology in emission permit markets with banking. Environ Resour Econ 22:369-390

Pizer WA (2002) Combining price and quality controls to mitigate global climate change. J Public Econ 85:409-434

Portney PR, Weyant JP (eds) (1999) Discounting and intergenerational equity. Resources for the Future, Washington, DC

Requate T (forthcoming) Commitment and timing of environmental policy, adoption of new technology, and repurcussions on R\&D. Environ Resour Econ

Requate T, Unold W (2003) Environmental policy incentives to adopt advanced abatement technology — will the true ranking please stand up. Eur Econ Rev 47:125-146

Rubio SJ, Casino B (2005) Self-enforcing international environmental agreements with a stock pollutant. Span Econ Rev 7:89-109

Ulph A, Ulph D (1997) Global warming, irreversibility and learning. Econ J 107:636-649

United Nations Third Conference of the Parties of the Framework Convention on Climate Change (1997) Kyoto protocol to the United Nations framework convention on climate change. United Nations

Weitzman ML (1998) Why the far distant future should be discounted at its lowest possible rate. J Environ Econ Manage 36:201-208 
von Weizsäcker CC (1971) Steady state capital theory. Springer, Berlin

Winkler R (2003) Zeitverzögerte Dynamik von Kapital- und Schadstoffbeständen. Eine österreichische Perspective (Time-lagged Dynamics of Capital Stocks and Stocks of Pollutants. An Austrian Perspektive). Metropolis, Marburg

Winkler R (2004) Time-lagged accumulation of stock pollutants. Additively separable welfare functions reconsidered. Discussion-Paper No. 408, Alfred Weber-Institute of Economics, University of Heidelberg

Winkler R (2005) Structural change with joint production of consumption and environmental pollution: a neo-Austrian approach. Struct Change Econ Dyn 16:111-135

Winkler R, Brand-Pollmann U, Moslener U, Schlöder J (2005) On the transition from instantaneous to timelagged capital accumulation. The case of Leontief-type production functions. Discussion-Paper No. 05-30, Centre for European Economic Research (ZEW), Mannheim

Winkler R, Brandt-Pollmann U, Moslener U, Schlöder JP (2004) Time lags in capital accumulation. In: Ahr D, Fahrion R, Oswald M, Reinelt G (eds) Operations research proceedings. Springer, Heidelberg

Wirl F (1995) The cyclical exploitation of renewable resource stocks may be optimal. J Environ Econ Manage 29:252-261

Wirl F (1999) Complex, dynamic environmental policies. Resour Energy Econ 21:19-41

Wirl F (2002) Stability and limit cycles in competitive equilibria subject to adjustment costs and dynamic spillovers. J Econ Dyn Control 26:375-398

Yang Z (2003) Reevaluation and renegotiation of climate change coalitions - a sequential closed-loop game approach. J Econ Dyn Control 27:1563-1594

van der Zwaan BCC, Gerlagh R, Klaassen G, Schrattenholzer L (2002) Endogenous technological change in climate change modelling. Energy Econ 24:1-19 